\title{
Dtmf Detection Using Goertzel Algorithm
}

\author{
${ }^{1}$ Sai Bharadwaj B, ${ }^{2}$ V.Sharmila Gowri, ${ }^{3}$ V.Bindu Madhuri \\ ${ }^{1,2,3}$ (ECE, Vignan's Institute of Engineering For Women,India)
}

\begin{abstract}
Dual-tone multi-frequency $(D T M F)$ is an international signaling standard for telephone digits(number buttons). These signals are used in touch-tone telephones as well as many other areas. Since analog devices are rapidly changing with digital devices, digital DTMF decoders become more important. The subject of this paper is to build a dual-tone multi-frequency (DTMF) signal detector. There are many algorithms for DTMF detection, and among all of them the chosen one is Goertzel's algorithm. It is one of the simplest algorithms of all and it is very often used in practical realizations. The simulation of this algorithm is done in MATLAB and outputs of this test are given in this paper.
\end{abstract}

Keywords: Pulse dialing,Tone dialing, Push button tone dialing,Digital frequency spectrum, Fast fourier spectrum, Goertzel algorithm, Energy threshold.

\section{Introduction:}

Pulse dialing and tone dialing are the two systems used in telephone signaling. DTMF is the name used for push-button tone dialing. DTMF uses two tones to represent each key on the touch pad. DTMF has found widespread applications such as in automated telephone inquiry services where a user can select options from a menu by sending DTMF signals from a telephone.

DTMF signaling represents a digit or a character by the sum of a high-frequency tone and a low frequency tone (thereby, dual tone). When any key is pressed, the tone of the column and the tone of the row are generated[5]. The DTMF corresponding to that key is generated by either table lookup or a resonator. The signal is transmitted through the telephone line to a switching center. The switching center samples the signal and uses FFT or Goertzel algorithm to determine the received frequencies and hence tell the corresponding digi[3].

\section{Methodology:}

Initially, a simple energy threshold is used to separate the digits in each file which can be done as follows. The whole signal is divided into frames whose individual average energy is calculated. The average of all these is considered as the threshold. But in some cases, the noise energy exceeds the threshold. Hence, five continuous frames are joined to make the noise energy less than the signal energy. This is done in steps 1 and 2 of the program. Now, the starting point of each digit is needed to be found, shown in step 3 . If the current frame number is 1 and the energy is more than the threshold, then it is one of the digit's starting points. For frame numbers after 1, if the frame energy is more than the threshold and also the previous frame energy, then it is again one of the digit's starting points. Then the Goertzel algorithm is implemented to obtain the frequency spectrum. Using the largest two amplitudes in the output of Goertzel algorithm, the digits in the signal are found.

\section{Understanding Fft And Goertzel Algorithm:}

In this as we have seen when a key is pressed a signal with corresponding two frequencies are transmitted. Now in order to carry out its functions and process the user's request, the public telephone system should first identify the dialed numbers. For this it carries the DTF and obtains the digital frequency spectrum. Depending upon the frequency content it identifies the number. The equations of DFT pair are shown below in equations (1) and (2):

$$
\begin{aligned}
& X(k)=\sum_{n=0}^{N-1} x(n) e^{-j 2 \pi n k / N}=\sum_{n=0}^{N-1} x(n) W_{N}^{n k} \quad \text { where } \mathrm{k}=0,1,2 \ldots \ldots . \mathrm{N}-1 \ldots \ldots \ldots . . . \\
& x(n)=\frac{1}{N} \sum_{k=0}^{N-1} X(k) e^{j 2 \pi n k / N}=\frac{1}{N} \sum_{k=0}^{N-1} X(k) W_{N}^{-n k} \text { where } \mathrm{n}=0,1,2 \ldots, \mathrm{N}-1 .
\end{aligned}
$$

Where $x(n)$ represents the discrete time signal, $X(k)$ represents discrete frequency signal and $\mathrm{N}$ is the number of points sampled in the frequency spectrum for the DFT analysis[1].

Comparison of time required for DFT, FFT, Goertzel algorithms. One important factor in determining whether the DFT can be applied efficiently is the time constraint involved. To be able to process the 'dial 
request', the above calculations should be as fast as possible. This is obvious from the fact that the computation of DFT involves complexity calculations (Eq 1). For one $\mathrm{k}, 4 \mathrm{~N}$ real multiplications are needed. Hence, computing $\mathrm{N}$ point $X(k)$ requires $4 N^{2}$ real multiplications. As such, if we had the value of $\mathrm{N}=1024$ with a computation speed of 40MIPS, the total number of multiplications is 4194304 . The time it would take for this is 4194304/40M which is approx. 0.1 seconds. For a complete phone number of length 10 digits, it would take a bit over 1s. Therefore, it isn't a very viable option to use in a real-life practical scenario.

A.Working of the FFT algorithm: The FFT algorithm requires only $2 N \log _{2} N$ real multiplications. Using the same example with $\mathrm{N}=1024$ and the computation speed as 40MIPS, the total number of multiplications is just 20480, and it takes 0.000512 seconds; which is a far better performance than $0.1 \mathrm{~ms}$ in the case earlier[2].

B.Working of the Goertzel Algorithm: : Goertzel algorithm[4] is especially useful when the number of frequency points needed to compute are less than N. If we have to evaluate M number of DFT coefficients, where $\mathrm{M}<2 \log _{2} N$ the number of real multiplications required for this algorithm is nearly equal to $\mathrm{M}(\mathrm{N}+2) \sim \mathrm{MN}$. With our typical values of $\mathrm{N}=1024$ and $\mathrm{M}=7$, the total number of multiplications needed is 7148 , which takes about 0.0001787 seconds; undoubtedly, the best among the three. By using the Goertzel method, maximum time utilization is obtained and the fastest response obtained.

The equations of the Goertzel algorithm are:

$$
\begin{aligned}
& v_{k}(n)=2 \cos \left(\frac{2 \pi}{N} k\right) v_{k}(n-1)-v_{k}(n-2)+x(n) \\
& y_{k}(n)=v_{k}(n)-\cos \left(\frac{2 \pi}{N} k\right) v_{k}(n-1)+j \sin \left(\frac{2 \pi}{N} k\right) v_{k}(n-1)
\end{aligned}
$$

1. Set $v_{k}(n)=0$ for $n<0$ and $v_{k}(n)=x(0)$

2. Iterate $(\mathrm{Eq} 3) \mathrm{N}$ times starting at $\mathrm{n}=1$

3. Use (Eq 4) at the end to find $y_{k}(N)$

This algorithm is used to check if the signal contains specific frequency components. In DTMF, we need to check 8 coefficients which correspond to the frequency values of $697,770,852,941,1209,1336,1477$ and $1633 \mathrm{~Hz}$ respectively, which are characteristic to the DTMF signaling being implemented.

\section{Results}

\section{A.Phone NumberA:}

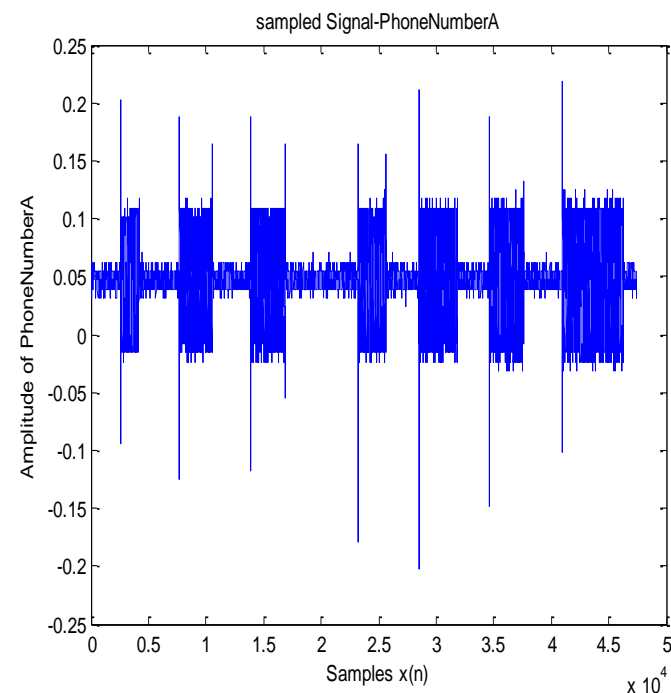

Fig 1.:Input signal plot

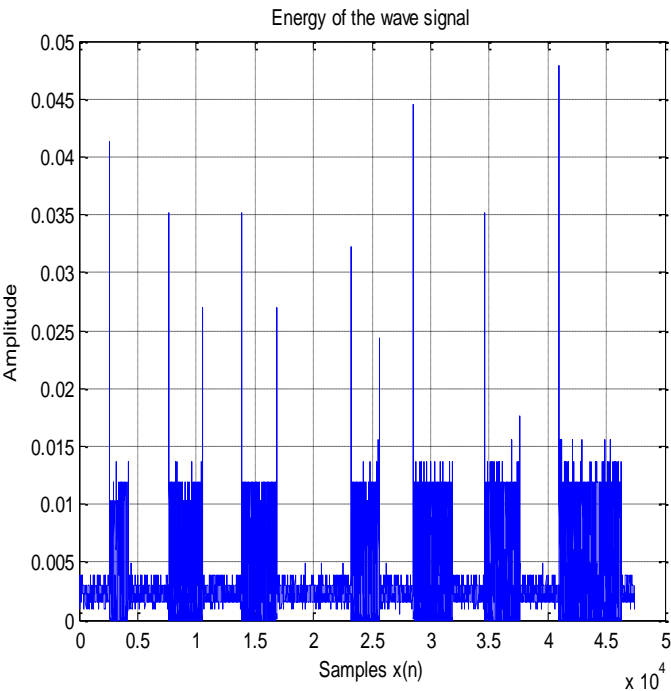

Fig 2.: Energy of the wave signal plot: 


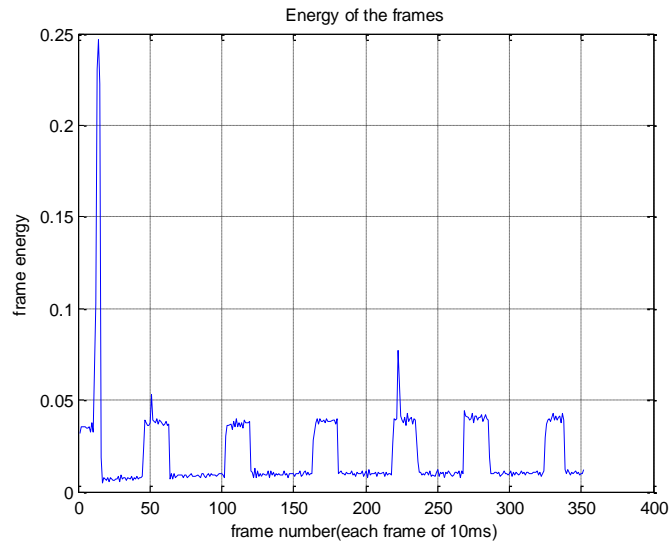

Fig 3.: Frame energies plot:

FFT OUTPUTS:
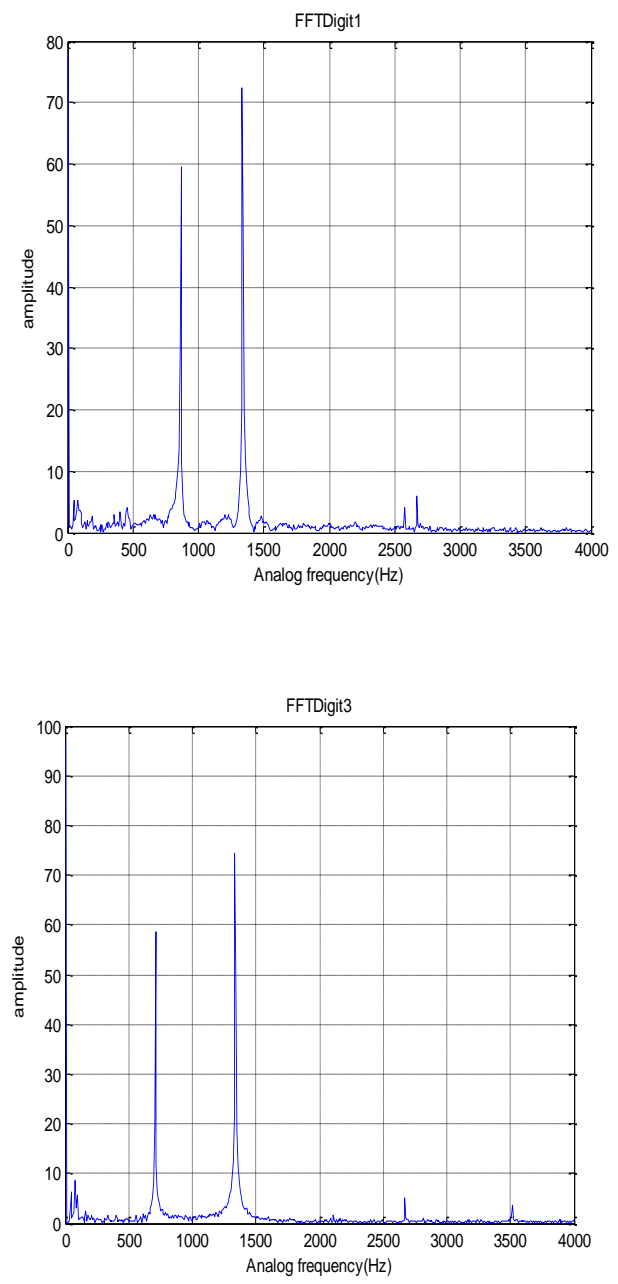

Fig 4.(c)FFT digit 3

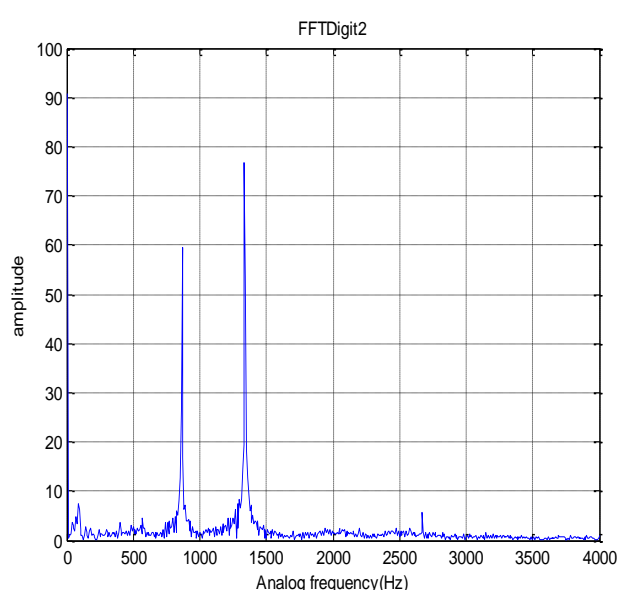

Fig 4.(a)FFT digit 1 4.(b)FFT digit 2

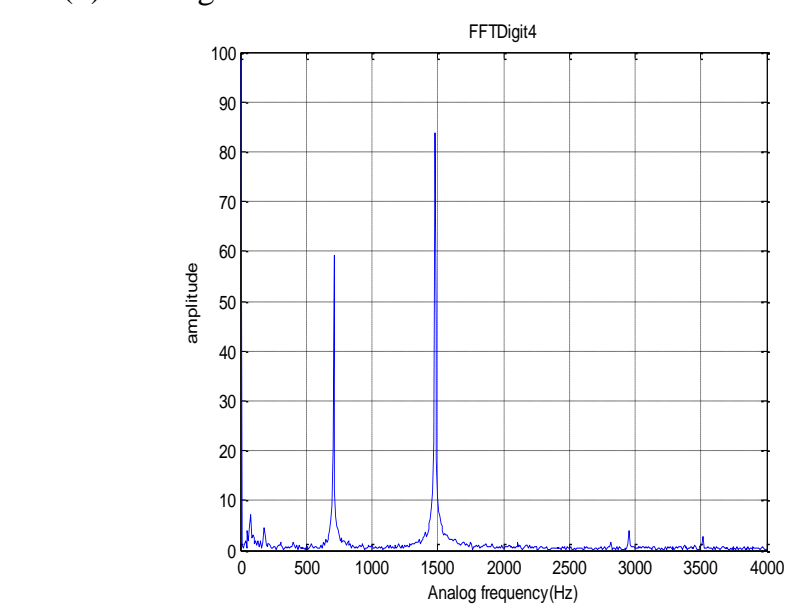

Fig 4.(d)FFT digit 4

Fig 

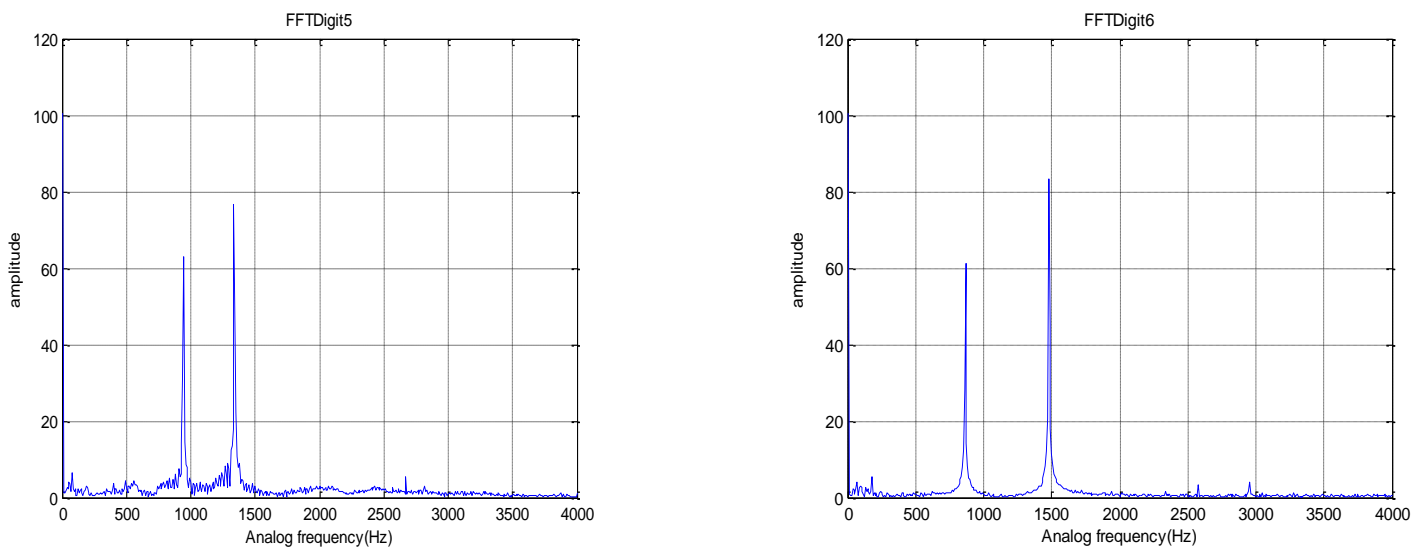

Fig 4.(e)FFT digit 5

Fig

4.(f)FFT digit 6

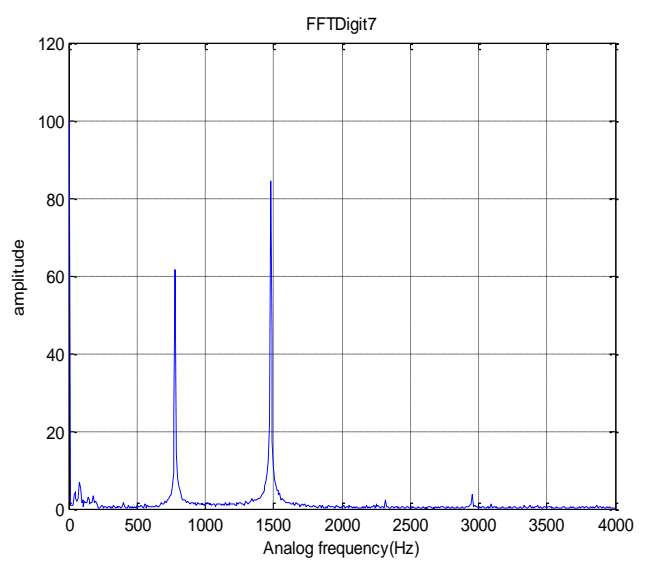

Goertzal Algorithm outputs:

Fig 4.(f)FFT digit 7

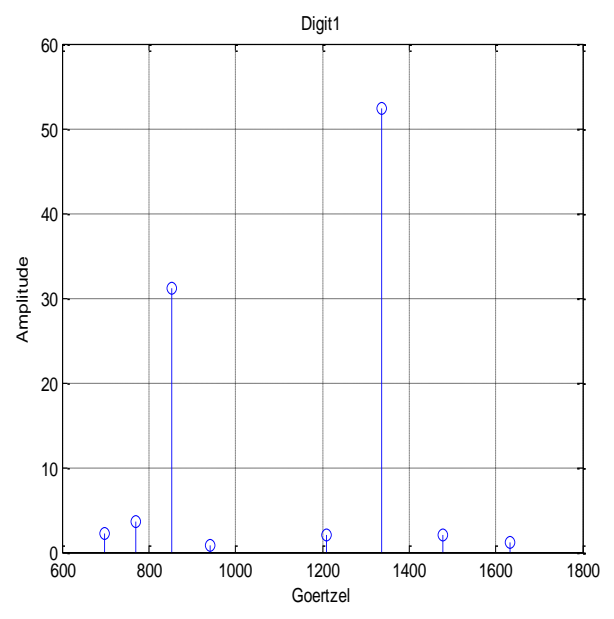

Fig 5.(a):digit 1

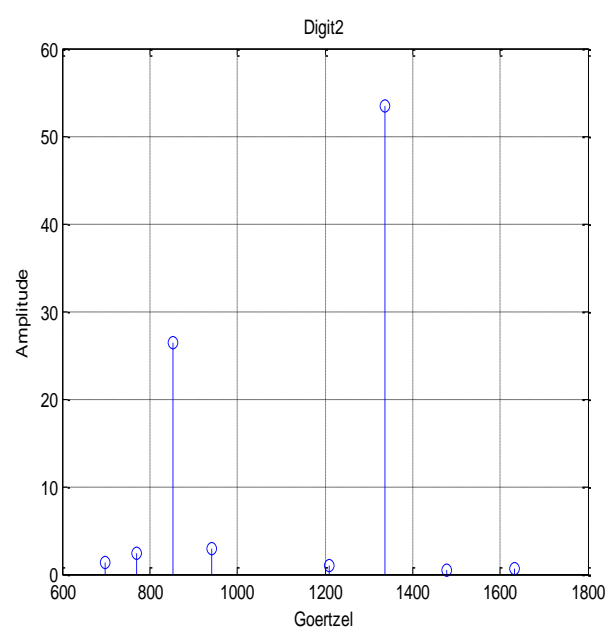

Fig 5.(b):digit 2 


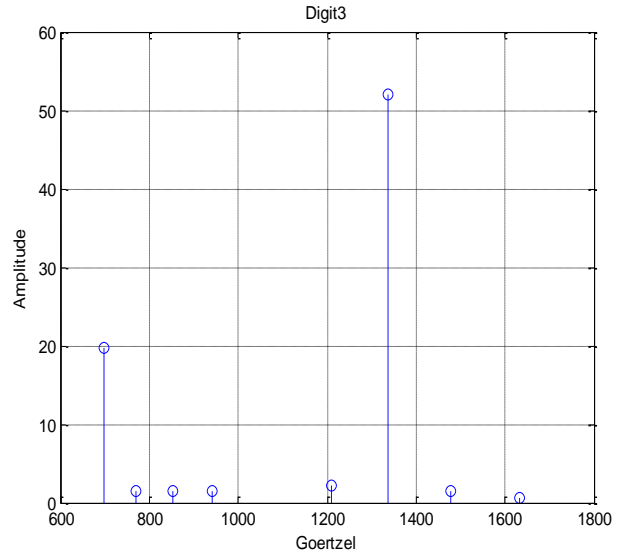

Fig 5.(c):digit 3

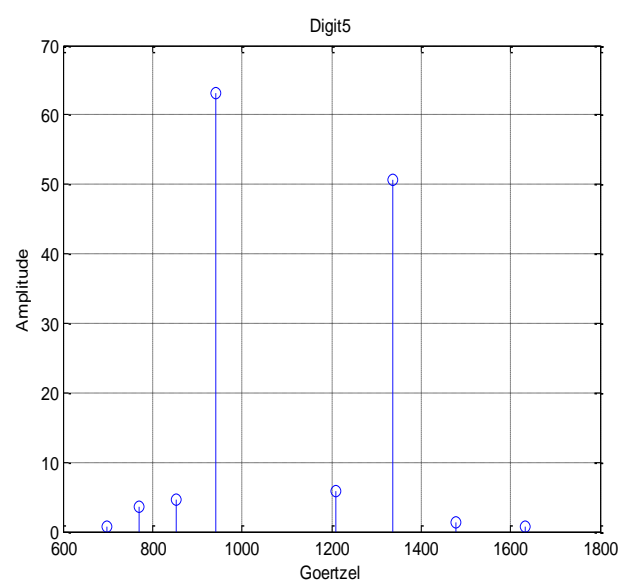

Fig 5.(e):digit 5

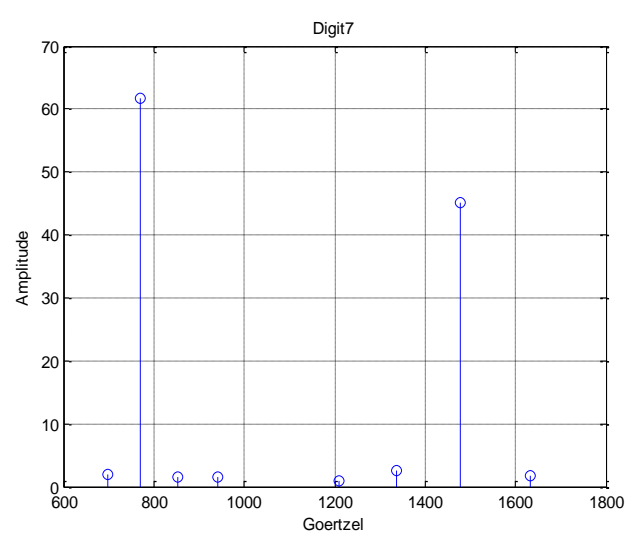

Fig 5.(g):digit 7

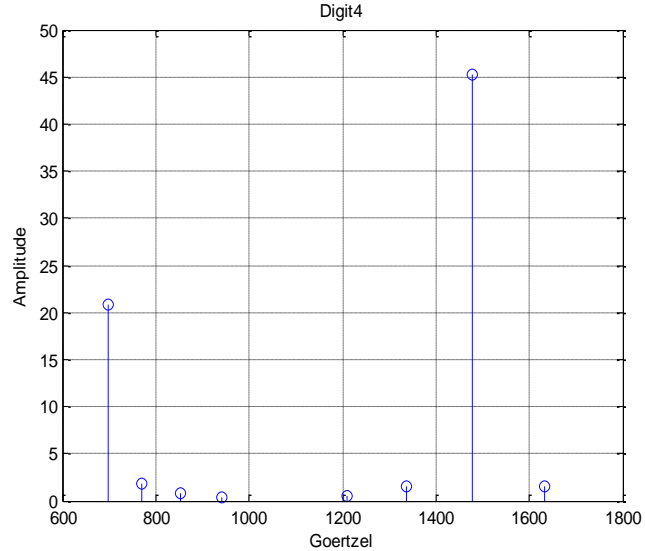

Fig 5.(d):digit 4

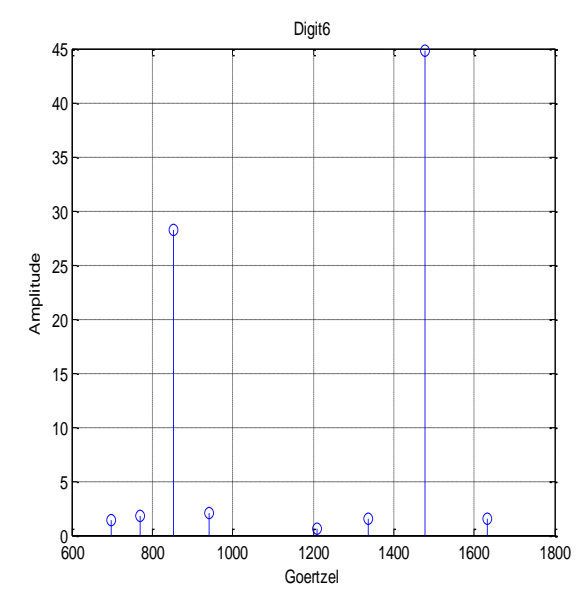

Fig 5.(f):digit6

\section{Output Of Phone Number}

Phone Number A = 8823096 
B.Phone NumberB:

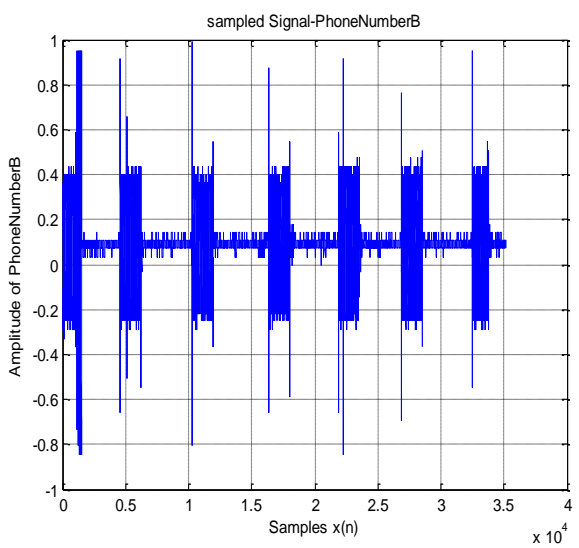

Fig .6: Input Sequence

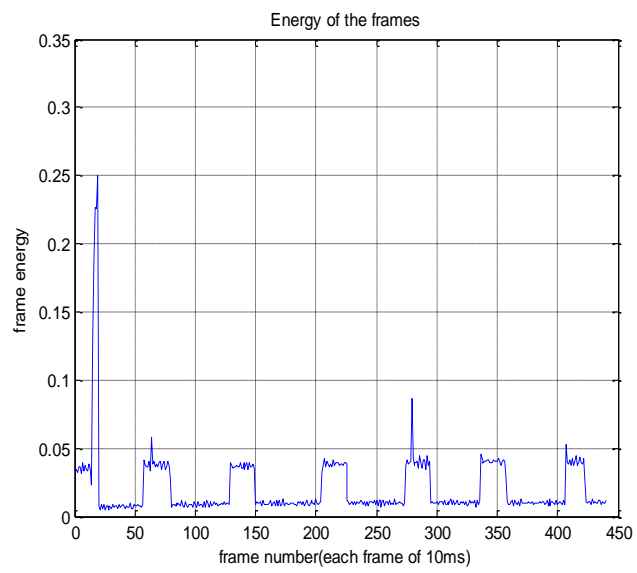

Fig .8:Frame energies plot

\section{FFT Outputs:}

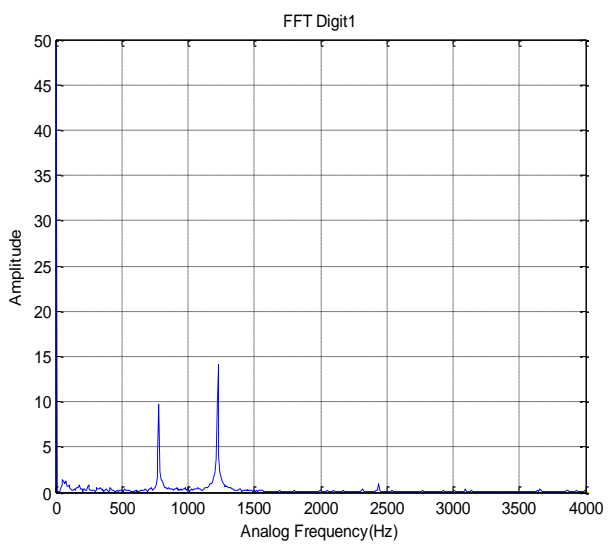

Fig.9(a):digit 1

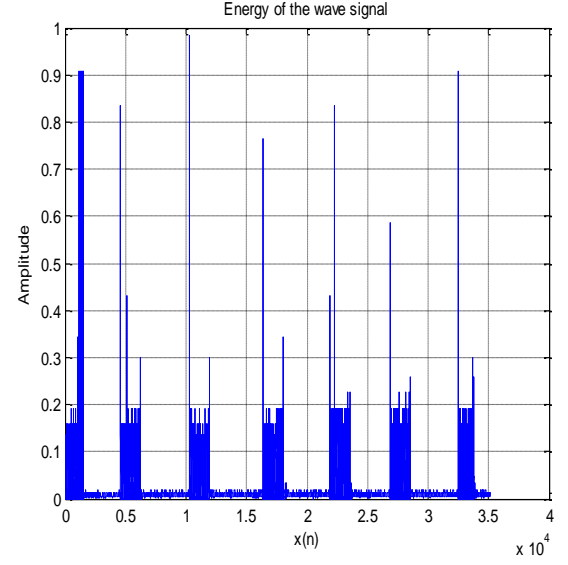

Fig.7:Energy of wave signal

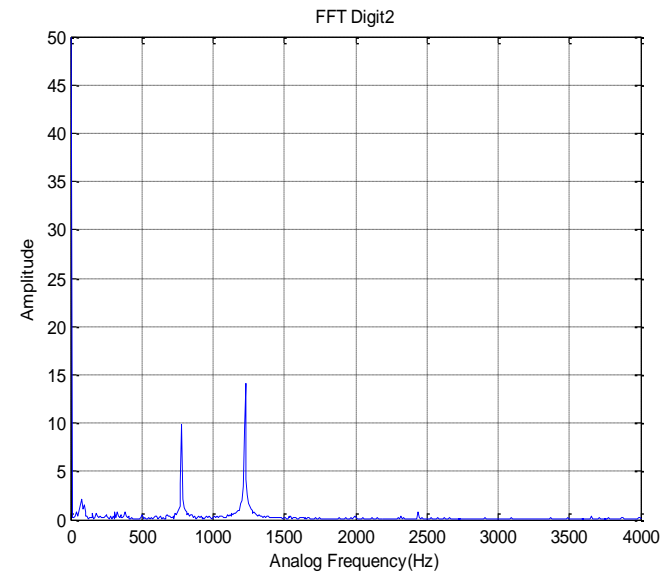

Fig.9(b):digit 2 


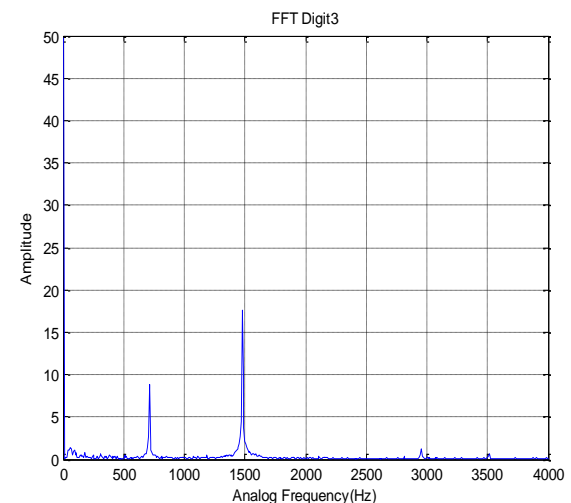

Fig.9(c):digit 3

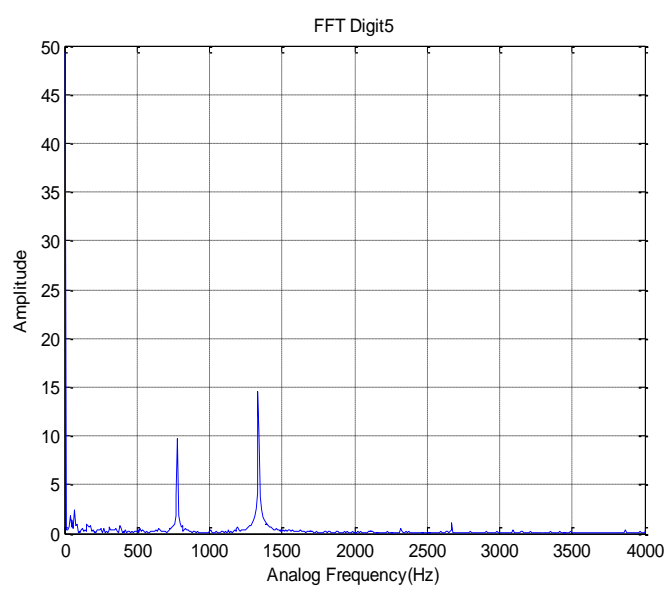

Fig 9.(e):digit 5

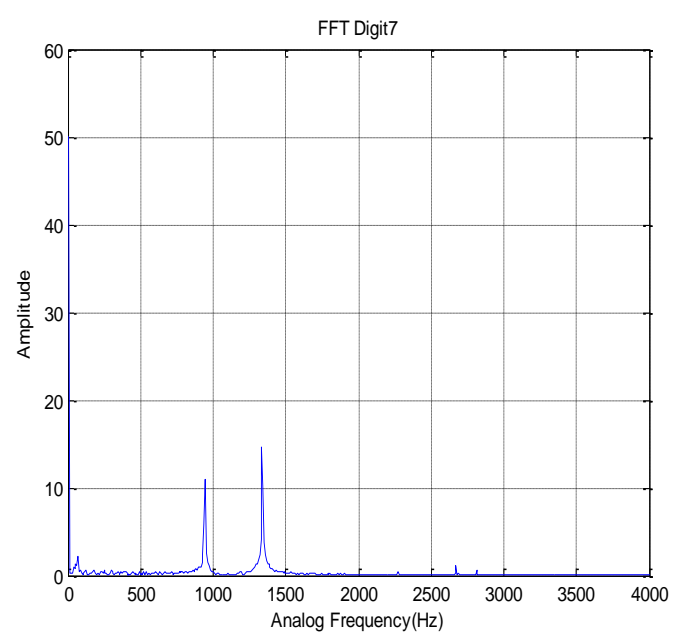

Fig.9(g):digit 7

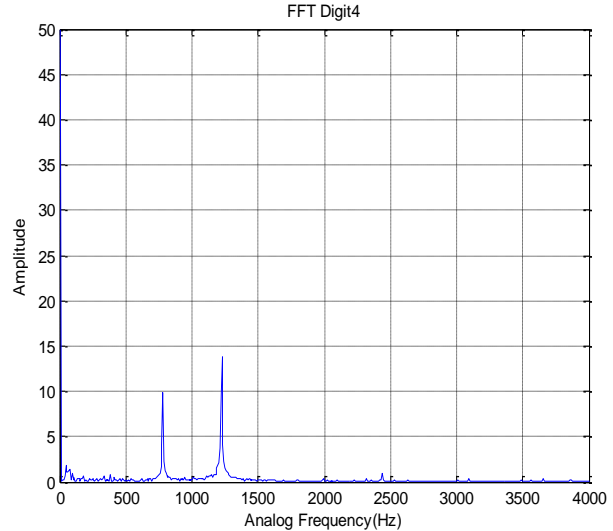

Fig.9(d):digit 4

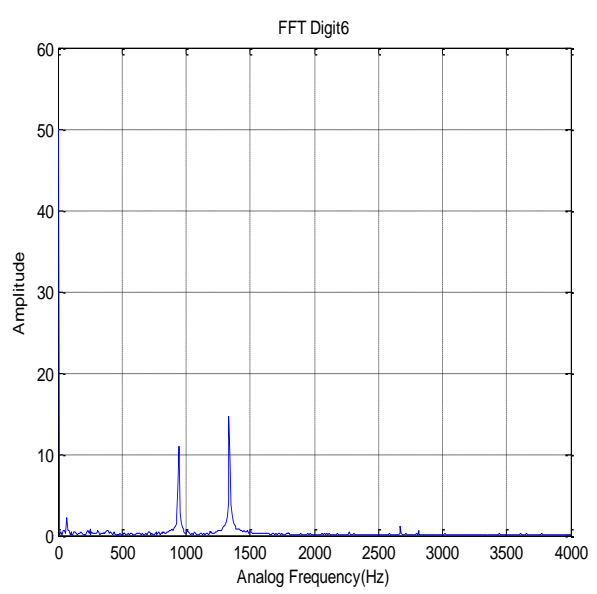

Fig.9(f):digit 6 
Goertzel Algorithm outputs:

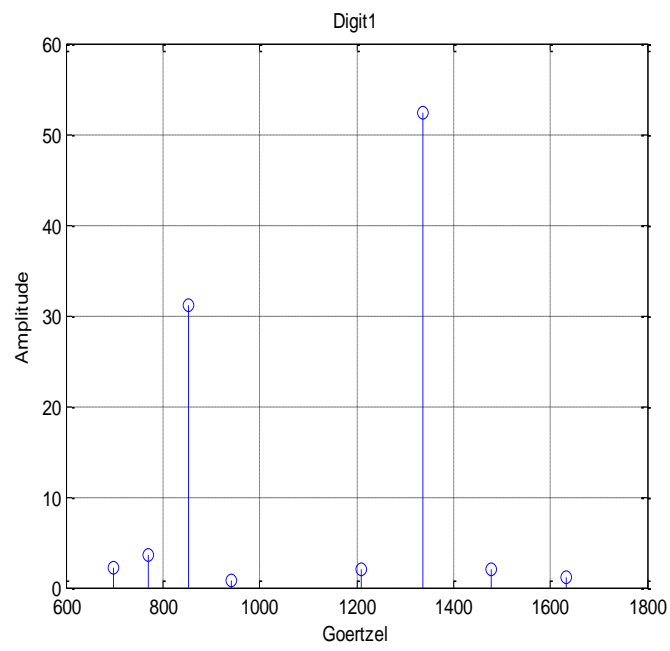

Fig.10(a):digit 1

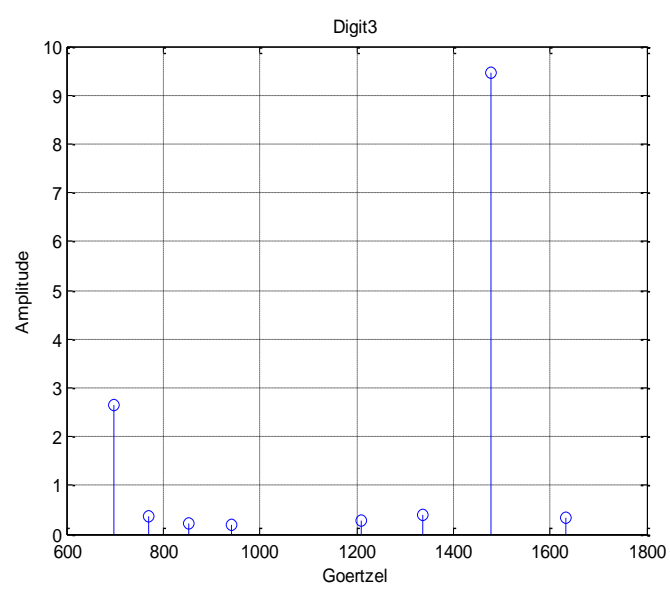

Fig.10(c):digit 3

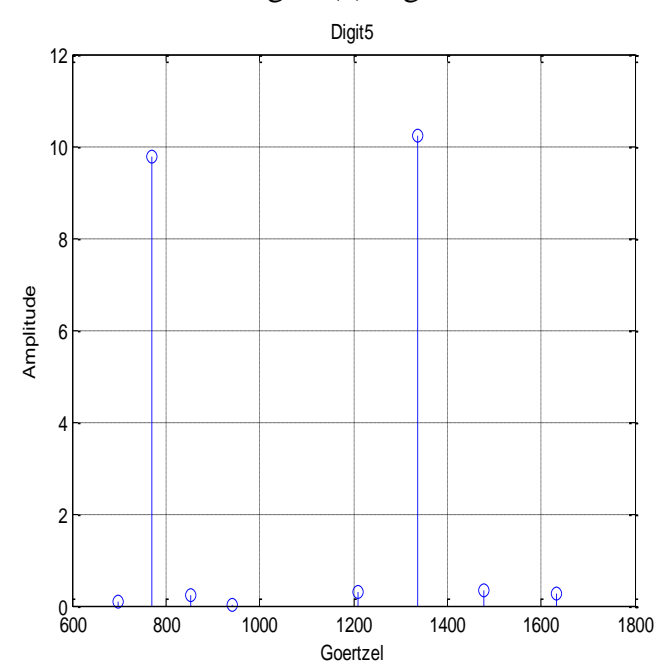

Fig.10(c):digit 5

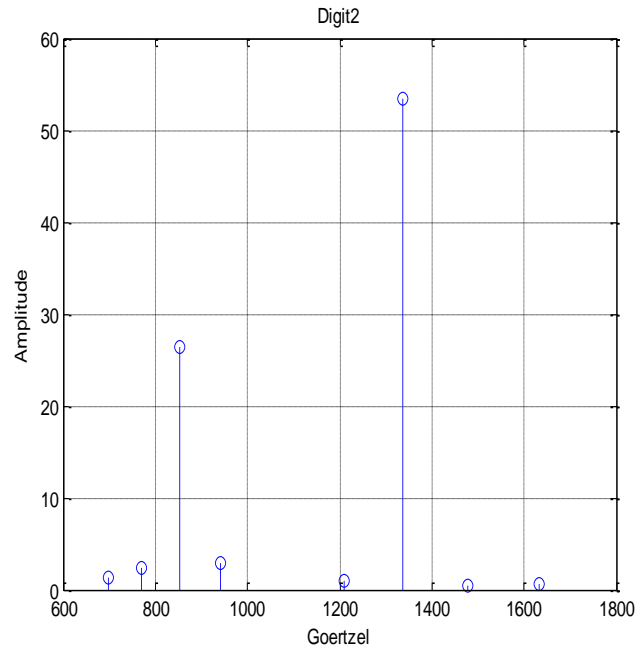

Fig.10(b):digit 2

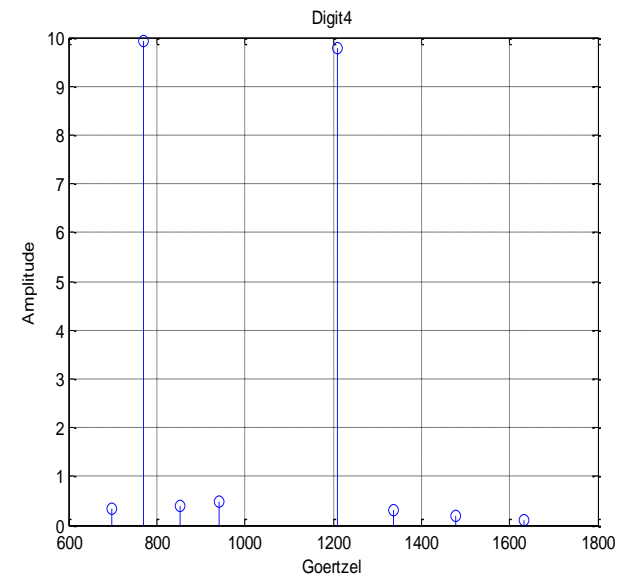

Fig.10(c):digit 4

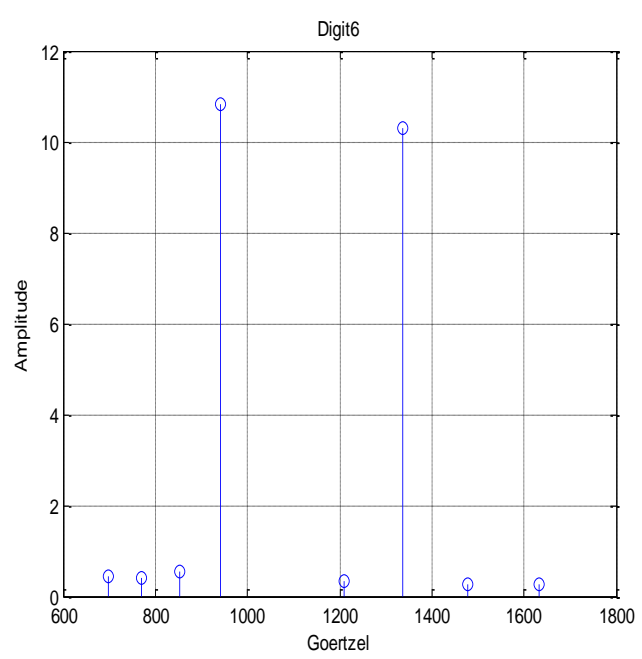

Fig.10(c):digit 6 


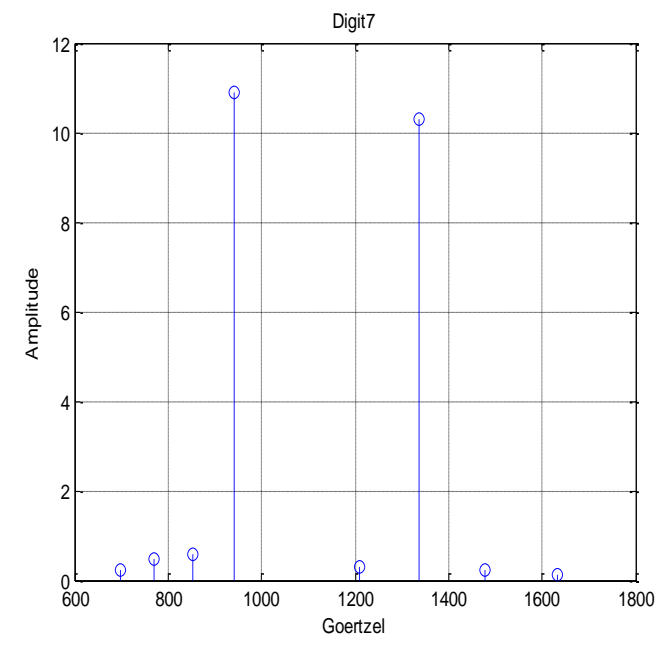

Output Of Phone Number:

Phone number $\mathrm{B}=4434500$

Fig.10(c):digit 7

\section{A. Location of the number digit:}

\section{Results And Discussion}

Energy threshold is an important parameter to determine the location of each digit number. The average energy of the frames is used as the threshold. In case the noise signal energy is higher than the threshold, which was the case in some plots; changing the frame size to $10 \mathrm{~ms}$ although it can be even more worked to smoothen out the noise. Therefore, the threshold can still be kept the same while accurately finding the 'start point'.

\section{B. FFT vs Goertzel method:}

By comparing the pairs of plots given the FFT and Goertzel methods, we see that for the two high peaks which we get for each digit we have higher amplitude for FFT computed peak compared to the Goertzel peak. This is might be because we are rounding off the K. Also if noise is effecting the signal FFt seems to accurate than the Goertzel. But for the purpose of detecting the digits Goertzel accuracy is sufficient as it give almost accurate frequencies if not exact ones.

As per the no. of computation we see that its greatly reduced when we use Goertzal as we calculate only 8 points with 1024 computations per point, compared to $20 * 1024$ in FFT. So there is a great speed up for Goertzal. This is an algorithm with a faster and equally efficient response.

Goertzel is much faster than FFT when $\mathrm{M}$ is smaller than $2 \log _{2} \mathrm{~N}$. In this project, we applied the Goertzel and FFT methods to tell the digit number contained in the wav file. Both methods get the same answer. However Goertzel method is an efficient way to analysis frequency spectrum at some fixed points as its faster.

\section{Observations}

Not many frequency points are required to decode the signal

1) If the Goertzel Algorithm criteria is satisfied, the time for computing is greatly reduced.

2) It is better than the FFT method.

\section{Conclusion}

The Phone numbers were detected, the threshold energy was set. The Phone numbers were detected using the Goertzel Algorithm and the FFT Technique.

PhoneNumberA $=8823096$

Phone number $\mathrm{B}=4434500$

Books:

[1]. RG Lyons, Understanding Digital Signal Processing, $2^{\text {nd }}$ edn $\quad$ [Prentice Hall PTR,NJ,2004].

Thesis:

[2]. Duhamel,MVetterli P,Fast Fourier transforms:A tutorial review and a state of the art.Signal process $19,259 \quad$ [1990]. Papers: 
[3]. SL Gay,J Hartung,GL Smith,Algorithms for multi-channel DTMF Detection for the WE DSP32 Family,IEEE on the proceedings of international conference on acoustics, Speech and Signal Processing [Glassglow,1989]

Websites:

[4]. Web:http://en.wikipedia.org/wiki/Goertzel_algorithm Q.23,Technical features of push-button Telephone sets [ITU-T,Geneva ],p, 1988 . 